\title{
CLIMA FAMILIAR Y AFRONTAMIENTO AL ESTRÉS EN PACIENTES ONCOLÓGICOS
}

\author{
FAMILY CLIMATE AND COPING WITH STRESS IN CANCER PATIENTS \\ Gloria Díaz A. ${ }^{1}$, Juan Yaringaño L. \\ Hospital Edgardo Rebaglati Martins de Lima, Perú \\ (RECIBIDO EL 08/02/2010, ACEPTADO EL 22/06/2010)
}

\begin{abstract}
RESUMEN
El presente estudio se orienta a establecer la relación entre el clima familiar y el afrontamiento al estrés en pacientes oncológicos del Hospital Edgardo Nacional Rebagliati Martins (HNERM). Se empleo el método descriptivo correlacional para describir y relacionar las dimensiones del clima familiar y los modos de afrontamiento de los pacientes oncológicos. La muestra estuvo conformada por 287 sujetos (hombres y mujeres), con promedio de 54 años, la mayoría casados, de instrucción técnica y superior. Se utilizó la Escala de Clima Social Familiar - FES de Moos (1982) y el Cuestionario de Afrontamiento al estrés en pacientes oncológicos - CAEPO de González (2004). Los resultados indican que la dimensión familiar Desarrollo se ve afectada significativamente debido a disminución de actividades sociales por la dedicación al cuidado del paciente. Además de relación significativa entre un Adecuado clima social familiar y el Afrontamiento Positivo, y entre Relaciones y Enfrentamiento y Lucha Activa, Autocontrol y Control Emocional. Por otro lado las mujeres tienden a usar estrategias activas cognitivas de reinterpretación positiva en mayor medida que los hombres, y las pacientes con Cáncer de Mama, usan estrategias Positivas de Afrontamiento al estrés en relación con otros tipos de cáncer, y los que padecen cáncer de tiroides, linfoma No Hodking y cáncer de próstata muestran más sentimientos de incertidumbre con respecto a la evolución y futuro de su enfermedad.
\end{abstract}

Palabras clave: Pacientes oncológicos, clima social familiar, modos de afrontamiento al estrés.

\begin{abstract}
Cancer is a disease that increasing twice in the last thirty years. The present investigation aims to establish the relationship between family environment and coping with stress in cancer patients Edgardo Rebagliati Martins Hospital (HERM). Bearing in mind of the impact that disease oncology has serious effects not only the patient but also in the family system.. Descriptive method was used to correlate with the aim of describing and relating the ways of coping and dimensions of family environment in patients with cancer in the first phase. The sample consisted of 287 subjects being most married people, technical and higher education, female and male and averaged of 54 years. Were used as tools Social Climate Scale Family - Moos FES (1982) and the Questionnaire of Coping to stress in cancer patients - CAEPO

1 Psicóloga Clinica del Servicio de Psicología del Hospital Edgardo Rebagliati Martins. E-mail: agdiaza@hotmail.com.
\end{abstract}


González (2004). The results indicate that the development dimension is affected to a greater extent due to the decline in social activities of the family for most of this dedication to patient care. A significant relationship was found, between family environmental suitable and positive coping, just as relations between the dimension relationship and Coping and Active Control, Self-control and emotional control. By taking into account gender differences is that women tend to use active cognitive strategies of positive reinterpretation greater extent than men, besides patients with Breast Cancer, use positive coping strategies to stress in relation to other types, and conversely those suffering from thyroid cancer, lymphoma No Hodking and prostate cancer not show feelings of uncertainty regarding the development and future of their disease.

Keywords: Cancer patients, family environmental and coping strategies.

\section{INTRODUCCIÓN}

El cáncer en la actualidad representa un problema de salud que demanda intervenciones prioritarias tanto en políticas de prevención, como en el diagnóstico y el tratamiento. Es una de las principales causas de muerte y discapacidad.

El paciente oncológico enfrenta múltiples problemas médicos, psicológicos y sociales, cuya complejidad aumenta con el tiempo y están directamente asociados a los diferentes estadios de la enfermedad y a los procesos terapéuticos. Así mismo está obligado a realizar ineludibles cambios individuales, familiares, laborales y sociales en muchos aspectos de su vida cotidiana, existiendo seria dificultad para sobrellevar esta enfermedad por lo que los modos de afrontamiento tienen un papel imprescindible que incidirá en la calidad de vida del paciente, al igual que el soporte familiar.

Cabe destacar que en las últimas décadas, los avances de la tecnología y en la medicina, permiten a las personas sobrevivir a enfermedades de curso fatal, con ello el incremento de sus posibilidades de vida y en mejores condiciones de salud. Pero paralelo a éste avance y el aumento de la esperanza de vida, surge el concepto de enfermedad crónica y entre ellos el cáncer (Guillem, 2002). El cáncer no es sólo una enfermedad crónica, que amenaza la vida del paciente por la idea de una muerte próxima e inminente , sino también al disminuir su capacidad de control sobre su vida y encontrarse regido por procedimientos que ayudan a su supervivencia, lo torna vulnerable a diferentes reacciones psicológicas incluyendo el miedo a la muerte, a la desfiguración, al abandono, a la ruptura de las relaciones, a la incapacidad, a la pérdida de la independencia personal y económica, así como a mecanismos de negación, reacciones de ansiedad y culpa.

Aproximadamente la mitad de los pacientes que sufren cáncer presentan trastorno adaptativo, seguido por el trastorno mixto ansioso depresivo y el episodio depresivo, los cuales se cree son reactivos al conocimiento del diagnóstico (Kaplan, 1996), por ser una enfermedad que se asocia al dolor y a la muerte. Ante esta situación atribulada, el paciente despliega una serie de esfuerzos tanto cognitivos como conductuales y dependerá del significado subjetivo que tiene para él la enfermedad y la realidad de la misma, para que la tensión se canalice positiva o negativamente, por ende el afrontamiento al estrés tiene un papel importante como componente mediador entre las circunstancias estresantes y la salud (Bayés, 1985). 
Las características que presentan el cáncer y su difícil pronóstico, regularmente relacionada a malignidad y sufrimiento, hacen que los pacientes y sus familias vivan una verdadera crisis que puede desorganizar a esta familia y al habitual ritmo de vida que tenían, centrándose ahora en atender a su paciente. Las respuestas de la familia a esa situación dependen de una estructura previa de funcionamiento, de las experiencias acumuladas de afrontamientos a eventos importantes y una serie de mitos, creencias y tradiciones que se desarrollan y establecen alrededor de la enfermedad que les ha tocado enfrentar. Pero no todas las familias y sus miembros responden de manera similar ante este acontecimiento, por lo que podemos observar en muchos casos una gran incapacidad de los miembros de la familia para ofrecer una respuesta adecuada a las múltiples demandas y necesidades del paciente. Regularmente esta incapacidad se produce porque los miembros del grupo familiar sufren un shock emocional agudo, que nos les permite afrontar adecuadamente el problema y se rinden ante la enfermedad, con el consiguiente sufrimiento para el paciente. En todos los casos el impacto emocional es muy fuerte, pero a pesar de ello algunas familias pueden enfrentar este problema y procurarles ambientes saludables a sus pacientes, que sin duda contribuyen decididamente a mejorar su calidad de vida.

Considerando lo antes dicho y apreciando el inmenso valor que para la práctica profesional adquiere la participación de la familia en el tratamiento del paciente oncológico, el propósito de la presente investigación es establecer la relación que puede existir entre el clima familiar y el afrontamiento al estrés en los pacientes oncológicos que nos debe servir para desarrollar un conjunto de alternativas que permitan al paciente y sus familias afrontar de mejor manera esta enfermedad.

\section{MÉTODO}

El método de investigación utilizado fue descriptivo correlacional, ya que tiene como propósito medir la relación que existe entre las variables Clima Familiar y Afrontamiento al Estrés, (Hernández; Fernández 1997).

\section{Sujetos}

La población objetivo la conformaron los pacientes oncológicos de ambos sexos, que pertenecen a diferentes grupo etareos, estado civil y de instrucción, que se encuentran en tratamiento en los Servicios de Ginecología Oncológica y Medicina Oncológica del Hospital Nacional Edgardo Rebagliati Martins. Según la oficina de estadísticas del referido hospital en el segundo trimestre del año 2009, asistieron 1000 pacientes a consulta externa con la patología antes mencionada. El número total de la muestra será de 287 pacientes, que cumplen con los criterios de inclusión, donde están representadas todas o casi todas las características de la población. De los cuales 106 fueron hombres y 181 mujeres, asimismo el $8 \%$ están entre 30 y 40 años, $28 \%$ entre 41 a 50 años, $28 \%$ entre 51 y 60 y $36 \%$ tiene más de 60 años. En la Tabla 1 se presentan los sujetos según el tipo de diagnóstico. 
Tabla N. ${ }^{0}$ 1. Descripción de los sujetos según tipo diagnóstico.

\begin{tabular}{lcc}
\hline & N & \% \\
\hline Cerviz & 13 & 4,5 \\
Colon & 8 & 2,8 \\
Estomago & 32 & 11,1 \\
Hígado & 6 & 2,1 \\
Leucemia & 28 & 9,7 \\
Linfoma no hodgking & 48 & 16,6 \\
Mama & 82 & 28,4 \\
Ovario & 11 & 3,8 \\
Piel & 10 & 3,5 \\
Próstata & 25 & 8,7 \\
Riñón & 4 & 1,4 \\
Testículo & 5 & 1,7 \\
Tiroides & 17 & 5,9 \\
\hline Total & 287 & 100,0 \\
\hline
\end{tabular}

\section{Instrumentos}

\section{Clima familiar: Escala de Clima Social Familiar (FES)}

Ficha técnica

Nombre original The Social Climate Scales: Family, work, Correctional Institutions and Classroom Envionment Scales

Autor R.H. Moos, B.S. Moos y E.J. Trickett

Procedencia Universidad de Stanford

Año 1982

Versión

Original en idioma Inglés.

Adaptación española $\quad$ : $\quad$ Fernández Ballesteros, R. y Sierra, B. 1984

Edad de aplicación : : Adolescentes y adultos.

Administración $\quad$ : $\quad$ Individual o colectiva

Duración $\quad$ : $\quad 20$ minutos aproximadamente

Adaptada al Perú por $\quad$ : $\quad$ César Ruiz Alva - Eva Guerra Turín - Lima 1993

\section{Descripción de la prueba}

Esta escala aprecia las características socio - ambientales de todo tipo de familias. Evalúa y describe las relaciones interpersonales entre los miembros de la familia, los aspectos de 
desarrollo que tienen mayor importancia en ella y su estructura básica. La escala consta de 10 sub escalas que definen tres dimensiones fundamentales:

- Relaciones: Es la dimensión que evalúa el grado de comunicación y libre expresión dentro de la familia y el grado de interacción conflictiva que la caracteriza. Esta integrada por tres sub-escalas: Cohesión, Expresividad y Conflicto.

- Desarrollo: Evalúa la importancia que tiene dentro de la familia ciertos procesos de desarrollo personal, que pueden ser fomentados o no, por la vida en común. Esta dimensión comprende las sub escalas de: Autonomía, Actuación, Intelectual - Cultural, Social - Recreativa y Moralidad - Religiosidad.

- Estabilidad: Proporciona información sobre la estructura y organización de la familia y sobre el grado de control que normalmente ejercen unos miembros de la familia sobre otros. La forman dos sub escalas: Organización y Control.

Puntuación. Las puntuaciones máximas son: 9 puntos en cada una de las 10 sub escalas. Normas: se utilizan normas "T" (Media: 50 - D.S.:10)

Tabla No 2. Niveles de clima social familiar.

\begin{tabular}{cl}
\hline T & \multicolumn{1}{c}{ Nivel } \\
\hline 70 a + & Significativamente alto \\
60 a 69 & Alto \\
41 a 59 & Promedio \\
31 a 40 & Bajo \\
30 a - & Significativamente bajo \\
\hline
\end{tabular}

\section{Confiabilidad}

Para la estandarización Lima, utilizando el método de Consistencia interna los coeficientes de confiabilidad van de $\mathrm{O} .80$ a 0.91 con una media de 0.89 para el examen individual, siendo las áreas de cohesión, intelectual - cultural, expresión y autonomía las más altas (la muestra usada para éste estudio fue de 139 jóvenes con promedio de edad de 17 años).

\section{Validez}

Se prueba el FES con la escala de TAMAI (Área familiar y al nivel individual) los coeficientes fueron en cohesión 0.62 , expresividad 0.53 y conflicto 0.59 . Éste trabajo demuestra la validez de la Escala del FES (la muestra individual fue de 100 jóvenes y de 77 familias) 


\section{Cuestionario de afrontamiento al estrés en pacientes oncológicos: CAEPO}

Ficha Técnica:

$\begin{array}{lll}\text { Autor } & : & \text { María Teresa Gonzáles Martínez } \\ \text { Procedencia } & : & \text { TEA Ediciones } \\ \text { Año } & : & 2004 \\ \text { Versión } & : & \text { Original en idioma castellano. } \\ \text { Edad de aplicación } & : & \text { Adultos que padecen cáncer } \\ \text { Administración } & : & \text { Individual } \\ \text { Duración } & : & 25 \text { minutos aproximadamente. }\end{array}$

\section{Descripción de la prueba}

Este cuestionario es un instrumento de recogida de información sobre las estrategias de afrontamiento (conductas y cogniciones) que utiliza un sujeto cuando se encuentra ante una situación de estrés, producida por el diagnóstico de su enfermedad, así como por las terapias y otras situaciones sociales y familiares que giran entorno a la enfermedad.

El cuestionario esta compuesto por 40 ítems distribuidos en siete escalas, cada una de las cuales corresponden a una determinada dimensión o estrategia de afrontamiento y se encuentran mezcladas para evitar la contaminación en las respuestas.

Las escalas son:

- $\quad$ Escala de Enfrentamiento y Lucha Activa (ELA): Indican que el sujeto valora la situación como grave y difícil pero, ve en ella un reto al que quiere responder con todos los recursos con los que cuenta.

- $\quad$ Escala de autocontrol y de Control Emocional (ACE): Indica que el sujeto dirige su comportamiento a hacer desaparecer o disminuir los estados afectivos displacenteros que le produce la enfermedad y lo que ésta conlleva.

- $\quad$ Escala de búsqueda de Apoyo Social (BAS): Indica que el sujeto considera importante para lograr un resultado positivo en torno a su recuperación, el apoyo afectivo, físico y moral de las personas cercanas a él.

- $\quad$ Escala de ansiedad y Preocupación Ansiosa (APA): Indica que el sujeto valora la situación como grave y muy amenazante, con incertidumbres respecto a la evolución y futuro de su enfermedad.

- $\quad$ Escala de pasividad y Resignación Pasiva (PRP): Indica que el sujeto considera que tiene muy pocas posibilidades para superar la situación grave, tiene una visión pesimista, y fatalista, respecto a su futuro, a la evolución y pronóstico de su enfermedad.

- Escala de huida y Distanciamiento (HD): Indican que el sujeto valora la situación como inadecuada, intentando escapar o eludir la realidad de la situación, hay un pesimismo extremo respecto a su pronóstico. 
- $\quad$ Escala de Negación (N): Indican que el sujeto niega la evidencia de la enfermedad y la realidad.

Tabla $\mathbf{N}^{0}$ 3. Tipos de afrontamiento.

\begin{tabular}{lc}
\hline Tipos de afrontamiento & Rango \\
\hline Positivo & $+3 \mathrm{a}+9$ \\
Preferentemente positivo & $+1 \mathrm{a}+2$ \\
No definido & 0 \\
Preferentemente negativo & $-1 \mathrm{a}-2$ \\
Negativo & $-9 \mathrm{a}-3$ \\
\hline
\end{tabular}

\section{Confiabilidad}

El estudio normativo se ha realizado con 160 sujetos que fueron diagnosticados de algún tipo de cáncer de cara y cuello. La confiabilidad se estableció mediante los coeficientes de consistencia interna (alfa de Cronbach), obteniéndose en todas las escalas índices superiores a 0,80 , excepto en la escala $\mathrm{N}$ donde la escala se acerca a ese valor.

\section{Validez}

La Validez de constructo se realizo mediante el análisis factoriales exploratorios (componentes principales con rotación varimax y criterio de Kaisser) se determinó 7 factores o dimensiones en el CAEPO. Los valores de las saturaciones factoriales se sitúan entre 0,64 y 0,87 , por lo que son muy satisfactorios y permiten afirmar la estructura factorial del cuestionario CAEPO y la validez de las escalas.

\section{Resultados}

\section{Fiabilidad de la Escala de Clima Social en la Familia}

En la tabla N. ${ }^{\circ} 4$, se observan que los subtest del FES presentan coeficientes de consistencia interna que varían de 0,970 a 0,992 y el test total presenta coeficiente de consistencia interna de 0,738 , lo que indica que los ítems del test tienen un comportamiento consistente. 
Tabla N. ${ }^{\circ}$ 4. Consistencia interna del FES, mediante el coeficiente Kuder Richardson.

\begin{tabular}{lc}
\hline Subtest & r20 \\
\hline Cohesión & 0,982 \\
Expresividad & 0,973 \\
Conflicto & 0,970 \\
Autonomía & 0,981 \\
Actuación & 0,984 \\
Intelectual cultural & 0,972 \\
Social recreativo & 0,992 \\
Moralidad religioso & 0,979 \\
Organización & 0,974 \\
Control & 0,973 \\
\hline Total & 0,738 \\
\hline
\end{tabular}

\section{Validez de constructo de la Escala de Clima Social en la Familia}

Observándose en la Tabla $\mathrm{N}^{0} 5$, que el coeficiente de correlación producto-momento de Pearson; de las 6 correlaciones posibles 5 son correlaciones estadísticamente significativas, siendo todas positivas. Es así, que las dimensiones: Relaciones, Desarrollo y Estabilidad presentan coeficientes de correlación de 0,$706 ; 0,562$ y 0,752 en cada caso; lo cual evidencia que el Clima Social Familiar en general, tiene validez del constructo.

Tabla N. ${ }^{\circ}$ 5. Coeficiente de correlación de Pearson de las dimensiones y total de la Escala de Clima Social Familiar.

\begin{tabular}{lcccc}
\hline & RELA & DESA & ESTA & FES \\
\hline Relaciones & 1 &, $125^{*}$ &, $362^{* *}$ &, $706^{* *}$ \\
Desarrollo & & 1 &, 064 &, $562^{* *}$ \\
Estabilidad & & & 1 &, $752^{* *}$ \\
FES & & & & 1 \\
\hline
\end{tabular}

Fiabilidad del Cuestionario de Afrontamiento al estrés para pacientes oncológicos

En cuanto a la fiabilidad se calculó mediante los coeficientes de consistencia interna de las escalas Alfa de Cronbach $(\alpha)$; para el caso del afrontamiento preferentemente Positivo $\alpha=0,642$ y en el caso del tipo del afrontamiento preferentemente Negativo $\alpha=0,709$. 
En la Tabla $\mathrm{N}^{\circ} 6$, figuran estos índices de cada una de las escalas del cuestionario según el tipo de afrontamiento. Vemos que en general presentan coeficientes de consistencia que van desde 0,427 hasta 0,683 .

Tabla N. ${ }^{\circ}$ 6. Consistencia interna del CAEPO, mediante el coeficiente Alfa de Cronbach.

\begin{tabular}{ccc}
\hline Sub escalas & $\mathbf{N}^{0}$ de ítems & Alfa de Cronbach \\
\hline ELA & 9 & 0,563 \\
ACE & 7 & 0,427 \\
BAS & 4 & 0,622 \\
APA & 6 & 0,594 \\
PRP & 5 & 0,634 \\
HD & 6 & 0,683 \\
N & 3 & 0,666 \\
\hline
\end{tabular}

\section{Validez del Cuestionario de Afrontamiento al estrés para pacientes oncológicos}

En primera instancia se llevó a cabo la validez discriminativa para lo cual se exploró las correlaciones entre las diferentes escalas y los modos de afrontamiento. De esta manera se observa en la Tabla $N^{0} 7$, que las subescalas enfrentamiento y lucha Activa (ELA), Autocontrol y Control Emocional (ACE), Búsqueda y Apoyo Social (BAS), correlacionan de manera directa y significativa con el modo de Afrontamiento Positivo al Estrés y en el caso de las escalas Ansiedad y Preocupación Ansiosa (APA), Pasividad y Resignación Pasiva (PRP) Huida y Distanciamiento (HD) y Negación (N), se observa que correlacionan de manera directa y significativa con el modo de Afrontamiento Negativo al Estrés, resultados que coinciden con la propuesta del Cuestionario de Afrontamiento al Estrés en Pacientes Oncológicos (CAEPO).

Tabla N. ${ }^{\circ}$ 7. Coeficiente de correlación de las escalas de Pearson y las estrategias positivas o negativas de afrontamiento al estrés.

\begin{tabular}{ccc}
\hline Escalas & Estrategias positivas & Estrategias negativas \\
\hline ELA &, $836^{* *}$ &,$- 495^{* *}$ \\
ACE &, $803^{* *}$ &,$- 463^{* *}$ \\
BAS &, $658^{* *}$ &,$- 275^{* *}$ \\
APA &,$- 551^{* *}$ &, $777^{* *}$ \\
PRP &,$- 385^{* *}$ &, $794^{* *}$ \\
HD &,$- 441^{* *}$ &, $668^{* *}$ \\
N &,$- 245^{* *}$ &, $687^{* *}$ \\
\hline
\end{tabular}

${ }^{*} \mathrm{p}<0,05 \mathrm{y} * \mathrm{p}<0,01$ 
Resultados inferenciales. Se establecieron las relaciones y diferencias entre las variables afrontamiento al estrés y clima familiar en pacientes oncológicos del Hospital Edgardo Rebagliti Martins.

En la Tabla N. ${ }^{\circ}$ 8, se observa la relación estadísticamente significativa entre el Afrontamiento Positivo las dimensiones del clima social familiar.

Tabla $N^{0}$ 8. Coeficiente Chi cuadrado $\left(\mathrm{X}^{2}\right)$ entre Afrontamiento Positivo y las dimensiones del Clima Social Familiar.

\begin{tabular}{lccc}
\hline & \multicolumn{3}{c}{ Afrontamiento positivo } \\
& $\mathrm{X}^{2}$ & $\mathrm{gl}$ & $\mathrm{p}$ \\
\hline Relaciones & 43,047 & 45 &, $045^{*}$ \\
Desarrollo & 57,209 & 36 &, $014^{*}$ \\
Estabilidad & 73,093 & 27 &, $000^{*}$ \\
\hline \multirow{2}{*}{$\mathrm{p}<0,05$} & & &
\end{tabular}

En la Tabla N. ${ }^{o} 9$ indica que existe relación estadísticamente significativa entre el Afrontamiento Negativo y las dimensiones del clima social familiar.

Tabla N. ${ }^{0}$ 9. Coeficiente Chi cuadrado $\left(\mathrm{X}^{2}\right)$ entre Afrontamiento Negativo y las dimensiones del Clima Social Familiar.

\begin{tabular}{lccc}
\hline & \multicolumn{3}{c}{ Afrontamiento negativo } \\
& $\mathrm{X}^{2}$ & $\mathrm{gl}$ & $\mathrm{P}$ \\
\hline Relaciones & 74,411 & 45 &, $004^{*}$ \\
Desarrollo & 51,656 & 36 &, $044^{*}$ \\
Estabilidad & 44,462 & 27 &, $019^{*}$ \\
\hline \multirow{2}{*}{$\mathrm{p}<0,05$} & & &
\end{tabular}

En la Tabla N. ${ }^{\circ}$ 10, se observa que existe relación significativa entre las estrategias ELA y ACE con la dimensión Relaciones del clima social familiar.

Tabla $N^{0}$ 10. Coeficiente Chi cuadrado $\left(\mathrm{X}^{2}\right)$ entre la Dimensión Relaciones y las Estrategias de Afrontamiento.

\begin{tabular}{cccc}
\hline & \multicolumn{3}{c}{ Relaciones } \\
& $\mathrm{X}^{2}$ & $\mathrm{gl}$ & $\mathrm{p}$ \\
\hline ELA & 34,542 & 25 &, $047^{*}$ \\
ACE & 49,943 & 30 &, $013^{*}$ \\
BAS & 37,165 & 35 &, 370 \\
APA & 44,240 & 35 &, 136 \\
PRP & 29,735 & 30 &, 479 \\
HD & 54,106 & 40 &, 064 \\
$\mathrm{~N}$ & 37,214 & 35 &, 367 \\
\hline \multirow{2}{*}{$\mathrm{p}<0,05$} & & &
\end{tabular}


En la Tabla N. ${ }^{\circ} 11$, se observa que existe relación significativa entre las estrategias ACE, BAS y APA, con la dimensión Desarrollo del Clima Social Familiar.

Tabla N. ${ }^{0}$ 11. Coeficiente Chi cuadrado $\left(\mathrm{X}^{2}\right)$ entre la Dimensión Desarrollo y las Estrategias de Afrontamiento.

\begin{tabular}{cccc}
\hline & \multicolumn{3}{c}{ Desarrollo } \\
& $\mathrm{X}^{2}$ & $\mathrm{gl}$ & $\mathrm{p}$ \\
\hline ELA & 26,585 & 20 &, 147 \\
ACE & 49,812 & 24 &, $001^{*}$ \\
BAS & 39,016 & 28 &, $041^{*}$ \\
APA & 43,382 & 28 &, $032^{*}$ \\
PRP & 31,529 & 24 &, 139 \\
HD & 34,629 & 32 &, 344 \\
N & 25,433 & 28 &, 604 \\
\hline
\end{tabular}

En la Tabla N. ${ }^{\circ} 12$, se observa que existe relación significativa entre las estrategias ELA, ACE, APA, PRP, HD y N con la dimensión Estabilidad del Clima Social Familiar.

Tabla N. ${ }^{0}$ 12. Coeficiente Chi cuadrado $\left(\mathrm{X}^{2}\right)$ entre la Dimensión Estabilidad y las Estrategias de Afrontamiento.

\begin{tabular}{cccc}
\hline & \multicolumn{3}{c}{ Estabilidad } \\
& $\mathrm{X}^{2}$ & $\mathrm{gl}$ & $\mathrm{p}$ \\
\hline ELA & 40,034 & 15 &, $000^{*}$ \\
ACE & 42,027 & 18 &, $001^{*}$ \\
BAS & 32,503 & 21 &, 052 \\
APA & 33,590 & 21 &, $040^{*}$ \\
PRP & 32,302 & 18 &, $020^{*}$ \\
HD & 37,648 & 24 &, $038^{*}$ \\
N & 34,040 & 21 &, $036^{*}$ \\
\hline
\end{tabular}

De acuerdo con la tabla N. ${ }^{\circ} 13$, se puede afirmar a partir de estos resultados que existe relación significativa de nivel medio entre las estrategias de Afrontamiento al Estrés y el Clima Social Familiar en pacientes oncológicos del Hospital Nacional Edgardo Rebagliati Martins.

Tabla N. ${ }^{\circ}$ 13. Coeficiente de correlación de Pearson entre las estrategias de afrontamiento y dimensiones de Clima Social Familiar.

\begin{tabular}{lcccc}
\hline & Relaciones & Desarrollo & Estabilidad & FES \\
\hline Positivas &, $459^{*}$ &,$- 499^{*}$ &, $548^{* *}$ &, $563^{*}$ \\
Negativas &,$- 451^{*}$ &, $431^{*}$ &,$- 489^{* *}$ &,$- 457^{*}$ \\
\hline$* . \mathrm{p}<0.05$ & & & & \\
$* * \mathrm{p}<0.01$ & & & &
\end{tabular}


La Tabla N. ${ }^{\circ} 14$ permite establecer que los pacientes oncológicos de sexo masculino y femenino presentan diferencias significativas en la dimensión Desarrollo

Tabla N. ${ }^{0}$ 14. Diferencias significativa en Clima Social Familiar según sexo.

\begin{tabular}{cccc}
\hline & Relaciones & Desarrollo & Estabilidad \\
\hline Mann-Whitney U & 9034,500 & 8341,500 & 8541,000 \\
Z & $-1,039$ & $-2,062$ & $-1,772$ \\
P &, 299 &, $039 *$ &, 076 \\
\hline
\end{tabular}

*. $\mathrm{p}<0.05$

De la Tabla N. ${ }^{\circ} 15$, podemos concluir que los pacientes oncológicos de sexo masculino y femenino presentan diferencias significativas en las estrategias de afrontamiento al estrés, salvo en Huida (HD).

Tabla N. ${ }^{\circ}$ 15. Diferencias significativas en Estrategias de Afrontamiento al Estrés, según sexo.

\begin{tabular}{cccccccc}
\hline & ELA & ACE & BAS & APA & PRP & HD & NEG \\
\hline Mann-Whitney U & 7,011 & 6,204 & 6,513 & 6,511 & 6,047 & 9,680 & 6,342 \\
Z & $-4,038$ & $-5,298$ & $-4,953$ & $-4,807$ & $-5,434$ &,- 084 & $-5,022$ \\
P &, $000 *$ &, $000 *$ &, $000 *$ &, $000 *$ &, $000 *$ &, 933 &, $000 *$ \\
\hline
\end{tabular}

*. $\mathrm{p}<0.05$

En la Tabla N. ${ }^{\circ}$ 16, se observa que los pacientes oncológicos presentan diferencias significativas en las estrategias Autocontrol y Control Emocional (ACE), Ansiedad y Preocupación Ansiosa (APA) y Pasividad y Resignación Pasiva y PRP, de afrontamiento al estrés de acuerdo con la edad.

Tabla N. ${ }^{0}$ 16. Diferencias significativas en Estrategias de afrontamiento, según edad.

\begin{tabular}{cccccccc}
\hline & ELA & ACE & BAS & APA & PRP & HD & NEG \\
\hline Kruskal-Wallis & 6,969 & 15,889 & 6,078 & 8,759 & 9,827 & 6,784 & 5,323 \\
Gl & 3 & 3 & 3 & 3 & 3 & 3 & 3 \\
P &, 073 &, $001 *$ &, 108 &, $033^{*}$ &, $020 *$ &, 079 &, 150 \\
\hline
\end{tabular}

*. $\mathrm{p}<0.05$

En la Tabla N. ${ }^{\circ} 17$ se observa que los pacientes oncológicos no presentan diferencias significativas en las dimensiones del Clima social Familiar según la edad. 
Tabla N. ${ }^{\circ}$ 17. Diferencias significativas en las dimensiones de Clima Social Familiar, según edad.

\begin{tabular}{cccc}
\hline & Relaciones & Desarrollo & Estabilidad \\
\hline Kruskal Wallis & 4,836 & 1,346 & 2,256 \\
\hline $\mathrm{gl}$ & 3 & 3 & 3 \\
\hline $\mathrm{p}$ &, 184 &, 718 &, 521 \\
\hline $\mathrm{p}>0,05$ & & &
\end{tabular}

La tabla N. ${ }^{\circ}$ 18, se determina que los grupos definidos por la variable tiempo de diagnóstico presentan diferencias significativas en las dimensiones del Clima Social Familiar.

Tabla $\mathbf{N}^{0}$ 18. Diferencias significativa en clima familiar según tiempo de diagnóstico en Clima social Familiar.

\begin{tabular}{cccc}
\hline & Relaciones & Desarrollo & Estabilidad \\
\hline Kruskal-Wallis & 6,167 & 6,870 & 20,818 \\
\hline $\mathrm{gl}$ & 4 & 4 & 4 \\
\hline $\mathrm{p}$ &, 187 &, 143 &, $000^{*}$ \\
\hline$* . \mathrm{p}<0.05$ & & &
\end{tabular}

La tabla N. ${ }^{\circ} 19$, se identifica la existencia de diferencias significativas entre el tiempo diagnóstico y las estrategias de afrontamiento, a excepción de los casos de BAS y N.

Tabla N. ${ }^{\circ}$ 19. Diferencias significativa en estrategias de afrontamiento según tiempo de diagnóstico, en Afrontamiento al estrés.

\begin{tabular}{cccccccc}
\hline & ELA & ACE & BAS & APA & PRP & HD & N \\
\hline Kruskal-Wallis & 11,791 & 10,891 & 3,427 & 14,732 & 19,491 & 12,861 & 5,284 \\
\hline $\mathrm{gl}$ & 4 & 4 & 4 & 4 & 4 & 4 & 4 \\
\hline $\mathrm{p}$ &, $019^{*}$ &, $028^{*}$ &, 489 &, $005^{*}$ &, $001^{*}$ &, $012^{*}$ &, 259 \\
\hline
\end{tabular}

*. $\mathrm{p}<0.05$

La tabla N. ${ }^{\circ} 20$, se observa que los grupos definidos por la variable tipo de cáncer la existencia de diferencias significativas según el tipo de cáncer y el clima familiar. 
Tabla N. ${ }^{0} 20$. Diferencias significativas en clima familiar según tipo de cáncer.

\begin{tabular}{cccc}
\hline & Relaciones & Desarrollo & Estabilidad \\
\hline Kruskal-Wallis & 23,633 & 12,069 & 23,392 \\
\hline $\mathrm{gl}$ & 5 & 5 & 5 \\
\hline $\mathrm{p}$ &, $000^{*}$ &, 034 &, $000^{*}$ \\
\hline
\end{tabular}

$*$. p $<0.05$

La tabla N. ${ }^{\circ} 21$, se observan diferencias significativas las estrategias de afrontamiento al estrés según tipo de cáncer; siendo los pacientes con cáncer de mama los utilizan en mayor medida la estrategias positivas de afrontamiento (ELA, ACE Y BAS); y por el contrario los pacientes con cáncer de tiroides, linfoma no Hodgkin y cáncer de próstata las estrategias negativas (APA, PRP., HD y N),

Tabla N. ${ }^{\circ}$ 21. Diferencias significativa en estrategias de afrontamiento según tipo de cáncer.

\begin{tabular}{cccccccc}
\hline & ELA & ACE & BAS & APA & PRP & HD & NEG \\
\hline Kruskal-Wallis & 40,587 & 44,489 & 13,583 & 115,892 & 138,663 & 40,568 & 112,839 \\
\hline Gl & 5 & 5 & 5 & 5 & 5 & 5 & 5 \\
\hline P &, $000^{*}$ &, $000^{*}$ &, $018^{*}$ &, $000^{*}$ &, $000^{*}$ &, $000^{*}$ &, $000^{*}$ \\
\hline
\end{tabular}

*. $\mathrm{p}<0.05$

\section{DISCUSIÓN}

Los resultados encontrados en el análisis psicométrico señalan que la Escala de Clima Social Familiar, presenta validez de constructo y confiabilidad por consistencia interna (Tablas N. ${ }^{\circ} 4,5$ y 6), del mismo modo Cuestionario de Afrontamiento al Estrés para pacientes oncológicos (Tablas N. ${ }^{\circ}$ 7, 8 y 9), al ser administradas en la muestra de pacientes oncológicos del Hospital Nacional Edgardo Rebagliati Martins.

En los resultados se observa que la dimensión Desarrollo que es un componente del Clima Social Familiar, es menor que las otras dos dimensiones (Estabilidad y Relaciones), lo cual se explica por la disminución de las actividades sociales de la familia al brindar mayor dedicación al cuidado del paciente; toda vez que el impacto emocional del diagnóstico y tratamiento que afronta el paciente, altera al sistema familiar, corroborándose lo señalado por Baider (2003).

Luego al analizar, la relación entre las dimensiones de la Escala de Clima Social Familiar y los modos de Afrontamiento al Estrés en pacientes oncológicos, se encontró la existencia de una relación significativa de modo directo, entre el Afrontamiento Positivo con las dimensiones del Clima Social Familiar, demostrándose que un buen Clima Social Familiar favorece el afrontamiento positivo al estrés. Confirmándose de este modo que el ajuste 
familiar frente al cáncer, puede percibirse como potencial de peligro de desintegración o como oportunidad para el fortalecimiento de la familia, recuperación, adaptación y comprensión de las necesidades y las expectativas de cada miembro de la familia, (Baider 1995).

Al analizar la relación entre la Dimensión Relaciones con la estrategias de afrontamiento al estrés en pacientes oncológicos, se encontró una relación significativa $(p<0,05)$ con Enfrentamiento y Lucha Activa; y Autocontrol y Control Emocional, información semejante a los resultados encontrados por Bartman y Cols (1996) sobre el uso; estrategias activas cognitivas de reinterpretación positiva, con el propósito de desarrollar una capacidad de ajuste más adaptado ante la enfermedad. En el caso de la dimensión Desarrollo del Clima Social Familiar se observa una relación significativa $(p<0,05)$ con Autocontrol y Control Emocional, Búsqueda de Apoyo social y Ansiedad y Preocupación Ansiosa; estos resultados refieren que los pacientes con cáncer, buscan dentro de sus procesos de desarrollo personal fomentados por la vida familiar, reducir la ansiedad y asumir una actitud optimista que permita reducir los estados displacenteros. En el caso de la dimensión Estabilidad se encontró relación significativa $(p<0,05)$ con las estrategias Enfrentamiento y Lucha Activa, Autocontrol y Control Emocional, Ansiedad y Preocupación Ansiosa, Pasividad y Resignación Pasiva, Huida y Negación. A diferencia de las otras dimensiones esta se relaciona con casi todas las estrategias de afrontamiento al estrés, a excepción de Búsqueda de Apoyo Social. Según estos resultados las familias de los pacientes oncológicos, se ven obligadas a reconstruir su estructura y organización, a modificar el grado de control que normalmente ejercen unos miembros de la familia sobre otros, en especial con el miembro familiar enfermo. El hecho que la búsqueda de apoyo social, no se relacione significativamente con la dimensión estabilidad, hace evidente que desde el conocimiento del diagnóstico la familia modifica sus relaciones para dar más atención al paciente. Luego durante el tratamiento la solución planificada de problemas parece ser la respuesta más efectiva ante situaciones de estrés de la familia, dado que se asocia a emociones resultantes más positivas tal como señalan Lázarus y Folkman (1986).

Al observar la relación entre las estrategias positivas de afrontamiento con las dimensiones del Clima Social Familiar, estas presentan una correlación positiva con la dimensión Relaciones y la dimensión Estabilidad, y por el contrario una correlación negativa con la dimensión Desarrollo; esto pone en claro que la libre expresión de emociones, el apoyo y compenetración de los miembros de la familia incrementan las estrategias positivas, por el contrario aquellas familias que favorecen la individualidad, la no dependencia de otros y falta de cohesión, tienden a utilizar estrategias negativas de afrontamiento al estrés.

En lo relacionado a diferencias en el Clima Social Familiar según el sexo del paciente se encontraron diferencias significativas a favor de las mujeres en la dimensión Desarrollo, no presentándose diferencias significativas en las dimensiones Relaciones y Estabilidad, esto parece indicar que en el caso de la paciente mujer, los procesos de desarrollo personal se mantienen en la vida en común y son estimulados, esto favorecería la autonomía y actuación, como señala Bartman y Cols (1996) las mujeres adultas mayores que tienden a usar estrategias activas cognitivas de reinterpretación positiva, por lo tanto su capacidad de ajuste es mayor al de los hombres, esto ultimo en concordancia con lo planteado por 
Blumberg el at. (1954) que administró el MMPI a pacientes masculinos con cáncer, y encontró que tenían mayores niveles de depresión, ansiedad, e inhabilidad para liberar o reducir el estrés.

El lo que respecta a las diferencias significativas según sexo, en las estrategias de afrontamiento al estrés, se encuentran diferencias significativas $(p<0,05)$, en todas las estrategias examinadas a excepción de Huida. Esto revela que las pacientes mujeres, al buscar la adaptación a la enfermedad, se organizan en tres grandes categorías: búsqueda de sentido, mantenimiento de la eficacia personal y construcción de la autoestima, tal como señala Kleinke (2001), favoreciendo el uso de estrategias positivas; pero en el caso de los hombres se aprecia el uso de estrategias negativas, indicando que los hombres tienden a sentir una mayor perdida de control de la situación con respecto al pronóstico de la enfermedad, así como un sentido fatalista y de negación de la realidad.

Al tomar en cuenta las diferencias en el uso de estrategias de afrontamiento según la edad del paciente se encuentran diferencias significativas $(p<0,05)$, en las estrategias Autocontrol y Control Emocional, Ansiedad - Preocupación Ansiosa y Pasividad - Resignación Pasiva. Estos resultados evidencian que los pacientes de 40 a 50 años logran disminuir los aspectos displacenteros de la enfermedad dirigiendo sus pensamientos hacia la evaluación y valoración de su situación personal. Por otro lado los pacientes de 30 a 40 años tienden a desarrollar mayores niveles de ansiedad y preocupación ansiosa, ante el cambio que representa la enfermedad oncológica, esto se relaciona con lo señalado por Folkman y Lazarus, (1988) quienes indican que el uso de la confrontación y el distanciamiento, resultaron ser estrategias menos efectivas para los problemas de adaptación ante la enfermedad en adultos entre 30 a 40 años. Por otro lado, en el Clima Social Familiar no se observan diferencias significativas entre las dimensiones según la edad.

Por el tiempo de diagnóstico, se encontraron diferencias significativas en las estrategias de afrontamiento, a excepción de los Búsqueda de Apoyo Social y Negación. Encontrándose que los pacientes con diagnóstico de 6 meses a 1 año, son los que mantienen mayor nivel en lucha activa y control emocional que los pacientes con diagnóstico de 1 a 3 años quienes presentan mayor preocupación ansiosa, resignación pasiva y negación, lo cual evidenciaría una perdida del optimismo ante el proceso de tratamiento, cuando este se prolonga más allá de un año.

Al analizar las diferencias significativas en el Clima Social Familiar según el tipo de cáncer, se observa un promedio más alto en la dimensión Relaciones en pacientes con Cáncer de Mama, seguido por el promedio de Estabilidad en pacientes con cáncer de próstata y por la dimensión Desarrollo en pacientes con Cáncer de Estómago.

En cuanto a las diferencias significativas en las estrategias de afrontamiento al estrés $(p<005)$. El rango de medias en pacientes con Cáncer de Mama, es mayor en las estrategias Positivas de Afrontamiento (Enfrentamiento y lucha activa, Autocontrol emocional y Búsqueda de apoyo social), mientras que los pacientes con cáncer de tiroides, linfoma no Hodgkin y cáncer de próstata presentan rangos de medias más altos en las estrategias negativas (Ansiedad y preocupación ansiosa, Pasividad y resignación pasiva, Huida y Negación). 
Esto indica que las pacientes con cáncer de mamá presentan una visión optimista con respecto a las posibilidades de lucha y pronóstico, desarrollando pensamientos que se dirigen a hacer desparecer estados afectivos displacenteros y una búsqueda de apoyo afectivo, físico y moral en las personas cercana a ellas, por el contrario los pacientes con cáncer de tiroides, linfoma No Hodking y cáncer de próstata, muestran sentimientos de incertidumbre con respecto a la evolución y futuro de su enfermedad, situación que los torna ansiosos y poco optimistas con falta de control en las situaciones estresantes que genera la enfermedad. Resultados similares a los de Carver y Cols. (1989) quienes evaluaron el rol mediador que tiene el afrontamiento sobre el optimismo en pacientes con cáncer de mama, evidenciando que la aceptación y el reencuadre así como el uso de la religión, fueron las estrategias de afrontamiento más usuales, mientras que la negación o la desinvolucración mental fueron poco usuales en éste grupo de pacientes.

\section{CONCLUSIONES}

De acerado con los resultados existe relación estadísticamente significativa entre el Clima Social Familiar y el Afrontamiento al Estrés.

Los pacientes oncológicos del Hospital Nacional Edgardo Rebagliati Martins, presentan en general un nivel Medio (Estable) en clima familiar, y en el caso del afrontamiento al estrés el promedio hace referencia a un afrontamiento positivo.

Existe relación significativa entre clima familiar y el afrontamiento positivo; se observa una relación significativa entre las dimensiones: Relaciones, Desarrollo y Estabilidad del clima social familiar con el Afrontamiento Positivo al estrés.

Existen diferencias significativas en los pacientes oncológicos varones y mujeres con respecto al clima familiar y el afrontamiento al estrés. Se observa que las mujeres que tiene estrategias de afrontamiento positivo en mayor medida en relación a los hombres.

No existen diferencias significativas entre los pacientes oncológicos de acuerdo a su edad, en función del clima familiar; sin embargo, existen diferencias significativas en estrategias de afrontamiento al estrés, siendo los pacientes de 40 a 50 años presentan afrontamiento positivo y los pacientes de 30 a 40 presentan afrontamiento negativo.

Existen diferencias significativas en los pacientes oncológicos de acuerdo al tiempo de diagnóstico, en función del clima familiar y afrontamiento al estrés; siendo los pacientes de 30 a 40 años los que presentan mayor Estabilidad en el clima familiar.

Existen diferencias significativas entre los pacientes oncológicos de acuerdo al tipo de cáncer, respecto al clima familiar. Los pacientes con cáncer de ovario presentan mayor Estabilidad en el clima familiar, los pacientes con cáncer de mama presentan estrategias de afrontamiento positivas, y por el contrario los pacientes con cáncer de tiroides, linfoma no Hodgkin y cáncer de próstata presentan un mayor promedio en estrategias negativas. 


\section{REFERENCIAS BIBLIOGRÁFICAS}

1. Astiz, J. (2001). Impacto psicológico del cáncer de mama en un medio rural. Revista de salud pública (de su versión virtual: salud pública.com).

2. Baraez V., M. (2002). Relación entre percepción de control y adaptación a la enfermedad en pacientes con cáncer de mama. Tesis para optar el grado académico de Doctora en Psicología, Universidad Autónoma de Barcelona, España.

3. Baider, L. (2003). Cáncer y familia: aspectos teóricos y terapéuticos. Revista Internacional de Psicológica Clínica y de la Salud, Vol. 3, $\mathrm{N}^{\circ}$ 1, pp. 505-520.

4. Bayés, R. (1994). Psicología oncológica. 2da ed. Edi. Martínez Roca S.A. Barcelona.

5. Bosch F. y Coleman M., P. (1992). Etiología del cáncer y epidemiología del cáncer. Manual de oncología Clínica Internacional. Unión Against Cancer. 5ta ed. Editorial Doyma Barcelona, España.

6. Cruzado y Olivares (1996). Intervención psicológica en pacientes con cáncer. Ed. Pirámide. Madrid.

7. Cruzado, J.A. y Labrador, F.J. (2000). Intervención psicológica en pacientes con cáncer. Revisiones en cáncer. Sran Ediciones, 14(2), 63-82.

8. Diccionario de especialidades oncológicas (2008). Lima: Thomson PLM Perú.

9. Elliot, G. \& Eisdorfer, C. (1982). Estrés humano y salud. Nueva York. Springer.

10. Ferrero, J. (1993). El afrontamiento de un diagnóstico de cáncer. Ed. Promolibro. Valencia.

11. Flórez Lozano J.A (1999). La comprensión del enfermo oncológico. En Eduardo García Camba. Manual de Psicooncología. Aula Médica, p. 171-190.

12. González Cajal J y Quesada Núñez G. (1999). Psicooncología y consentimiento informado. En Manual de psicooncología. Edic. Aula Médica, p. 401-414

13. González Barón M. (1999). La información al paciente con cáncer. Fundamentos. En Manual de Psicooncología. Edic. Aula Médica, p. 159-170.

14. González Barón M y Muñoz Sánchez JD (2002). La relevancia de la relación médico-paciente en la práctica clínica oncológica. En La relación médico-paciente en oncología. Una visión sociológica. Ars Medica, p. 1-10.

15. González M., M.T. (2004). Cuestionario de afrontamiento al estrés para pacientes oncológicos. TEA ediciones S.A. Madrid.

16. Ruiz-Benitez de Lugo Comyn, M.A. (2007). La conspiración del silencio en los familiares de los pacientes terminales. Tesis Doctoral. Facultad de Psicología. Universidad de La Laguna.

17. Urquidi, L.E. y Martiel, M.M. (2001). El cáncer como enfermedad multideterminada. Universidad Sonora, México: Manuscrito. 